\title{
Isotropy theorem for cosmological vector fields
}

\author{
J. A. R. Cembranos, C. Hallabrin, A. L. Maroto, and S. J. Núñez Jareño \\ Departamento de Física Teórica I, Universidad Complutense de Madrid, E-28040 Madrid, Spain \\ (Received 10 April 2012; published 10 July 2012)
}

\begin{abstract}
We consider homogeneous Abelian vector fields in an expanding universe. We find a mechanical analogy in which the system behaves as a particle moving in three dimensions under the action of a central potential. In the case of bounded and rapid evolution compared to the rate of expansion, we show-by making use of the virial theorem - that for an arbitrary potential and polarization pattern, the average energy-momentum tensor is always diagonal and isotropic despite the intrinsic anisotropic evolution of the vector field. For simple power-law potentials of the form $V=\lambda\left(A^{\mu} A_{\mu}\right)^{n}$, the average equation of state is found to be $w=(n-1) /(n+1)$. This implies that vector coherent oscillations could act as natural dark matter or dark energy candidates. Finally, we show that under very general conditions, the average energy-momentum tensor of a rapidly evolving bounded vector field in any background geometry is always isotropic and has the perfect fluid form for any locally inertial observer.
\end{abstract}

DOI: 10.1103/PhysRevD.86.021301

PACS numbers: $98.80 . \mathrm{Cq}$

Our knowledge about the history of our universe has improved over the last few years with the advent of a large amount of new observations. There are robust astrophysical data that support the existence of an early inflationary era; an additional matter component supplementing the baryonic one, known as dark matter; and a present era of accelerated expansion driven by dark energy. However, the fundamental nature of these components remains unknown. Different phenomenological and model building strategies have been proposed to understand them, in which rapidly evolving fields could play an important role.

In particular, different scalar dynamics have been considered as the possible answers to these open questions; standard inflation models are based on the slow-roll evolution of a scalar field known as inflaton. The dynamics of this field is able to successfully finish the accelerated regime, and its later rapid oscillations provide a mechanism for reheating, transferring perturbatively or nonperturbatively the energy density of the oscillations to the matter fields. Also, the possibility of generating inflationary expansion during the period of inflaton oscillations has been studied in [1]. Another example of rapidly evolving scalar fields can be found in nonthermal dark matter candidates like the axion [2] or other massive scalar [3] or pseudoscalar particles [4], in which the energy density of rapid scalar coherent oscillations scales precisely as nonrelativistic matter [5]. Dark energy models based on the dynamics of scalar fields are commonly known as quintessence. Oscillating evolutions have also been considered within this context [6].

On the other hand, it is interesting to remark that all these possibilities could in principle be offered by any bosonic degree of freedom and not only by scalars. In fact, a large number of fundamental vector fields are present in the standard model of particles and interactions, and in a large portion of its extensions. Therefore it is natural to consider vector models which could shed light on the above mentioned open problems in cosmology. However, there is an important distinctive feature of vector fields as compared with scalar fields. Even in a homogeneous configuration, vector dynamics is generally anisotropic.

Most parts of observational data seem to be consistent with an early homogenous and isotropic universe. Models supporting a large amount of anisotropy suffer severe constraints. However, despite this fact, there are some examples in which vector fields have been shown to provide interesting models in different cosmological scenarios. For instance, models of inflation based on vector fields have been extensively studied recently [7], and a first proposal for vector inflation which could avoid the generation of an excess of anisotropy can be found in [8]. Models of dark energy based on massive vector fields have also been considered in [9]. Vector dark energy without potential terms has been proposed in [10]. Vector models for dark matter based on hidden sector gauge bosons have been discussed in [11]. A possible role in the generation of metric perturbations in the so-called curvaton scenario has also been considered for vectors in [12]. In that work, it was shown that an oscillating massive homogeneous vector field behaves as nonrelativistic matter with an equation of state $\omega=0$, in a completely analogous way to the scalar case. Despite the anisotropy of the oscillations, the average energymomentum tensor (EMT) turned out to be isotropic in that case. This fact has been used in order to propose oscillating massive vector fields as nonthermal dark matter candidates in [13].

On the other hand, the potential of anisotropic cosmologies have received important attention in recent years, motivated mainly by the possible existence of anomalies in the isotropy of the cosmic microwave background (CMB) and matter distributions, which could be pointing to the existence of a preferred spatial direction in the universe [14].

In this work, we show that despite its intrinsically anisotropic evolution, the average EMT associated with 
rapidly evolving vector fields is isotropic under very general and natural conditions. The proof has a mechanical analogy with the virial theorem, and applies to a general Abelian gauge vector with any general self-interaction given by a potential of the form $V\left(A_{\mu} A^{\mu}\right)$.

In order to simplify the argument, let us consider first a flat Friedmann-Lemaître-Robertson-Walker (FLRW) metric, given by

$$
d s^{2}=d t^{2}-a^{2}(t) d \vec{x}^{2} .
$$

The Lagrangian density for a vector field with a potential which is an arbitrary scalar function of $A^{2}=A_{\mu} A^{\mu}$ is given by

$$
\mathcal{L}=-\frac{1}{4} F_{\mu \nu} F^{\mu \nu}-V\left(A^{2}\right),
$$

where, for an Abelian field, the field strength tensor is

$$
F_{\mu \nu}=\partial_{\mu} A_{\nu}-\partial_{\nu} A_{\mu} .
$$

The corresponding field equations read

$$
F_{; \nu}^{\mu \nu}+2 V^{\prime}\left(A^{2}\right) A^{\mu}=0,
$$

where $V^{\prime}(x)=d V / d x$. We also need to calculate the EMT:

$$
\begin{aligned}
T^{\mu}{ }_{\nu}= & \frac{1}{4} F_{\rho \lambda} F^{\rho \lambda} g^{\mu}{ }_{\nu}-F^{\rho \mu} F_{\rho \nu}+V\left(A^{2}\right) g^{\mu}{ }_{\nu} \\
& -2 V^{\prime}\left(A^{2}\right) A^{\mu} A_{\nu} .
\end{aligned}
$$

Assuming homogeneity for the vector field, i.e., $A_{\mu}=\left(A_{0}(t), A_{i}(t)\right)$, we obtain for the temporal component

$$
V^{\prime}\left(A^{2}\right) A_{0}=0,
$$

whereas for the spatial components, we get

$$
\ddot{A}_{i}+H \dot{A}_{i}-2 V^{\prime}\left(A^{2}\right) A_{i}=0 .
$$

The EMT components read:

$$
\begin{gathered}
\rho \equiv T_{0}^{0}=\frac{1}{2} \frac{\dot{A}_{i} \dot{A}_{j} \delta^{i j}}{a^{2}}+V\left(A^{2}\right), \\
p_{k} \equiv-T_{k}^{k}=\frac{1}{2} \frac{\dot{A}_{i} \dot{A}_{j}}{a^{2}} \delta^{i j}-\frac{\dot{A}_{k} \dot{A}_{k}}{a^{2}}-V\left(A^{2}\right) \\
-2 V^{\prime}\left(A^{2}\right) \frac{A_{k} A_{k}}{a^{2}}, \quad k=1,2,3, \\
T_{j}^{i}=\frac{T_{i}^{i}{ }_{0}=0,}{a^{2}}+2 V^{\prime}\left(A^{2}\right) \frac{A_{i} A_{j}}{a^{2}}, \quad i \neq j .
\end{gathered}
$$

Notice that in the definition of the pressures $p_{k}$, no summation in $k$ is assumed.
Now we can express the conservation law $T_{; \nu}^{\mu \nu}=0$ as

$$
\dot{\rho}+H\left(\sum_{k} p_{k}+3 \rho\right)=0 .
$$

We see that the off-diagonal part of the EMT does not contribute in Eq. (12) because of the homogeneity of the vector field.

The temporal equation Eq. (6), implies ${ }^{1}$

$$
A_{0}=0 \text {. }
$$

Therefore the homogeneity condition reduces the problem to the evolution of a 3 -vector $\vec{A}(t)$. We can define the typical time evolution scale of the spatial component $A_{i}$ from $\omega_{i} \sim\left|\dot{A}_{i} / A_{i}\right|$. This value coincides with the frequency for an oscillatory movement. In the case of rapid evolution of $A_{i}$ in relation to the universe expansion $\omega_{i} \gg H$, we can neglect time derivatives of the scale factor, so that defining $r_{i}=A_{i} / a$ in a time range of order $\omega_{i}^{-1}$, we have

$$
\dot{r}_{i}=\frac{\dot{A}_{i}}{a}-H r_{i} \approx \frac{\dot{A}_{i}}{a} .
$$

Thus we can ignore the friction term, and we see that Eq. (7) reduces to the evolution equation of a point particle with position vector $\vec{r}=\vec{A} / a$ in the presence of a central potential $V\left(-r^{2}\right)$. In particular, this implies that we can make use of the standard classical mechanics results. Thus, since the potential is central, we will have conservation of the corresponding angular momentum $\vec{L}=\vec{r} \times \dot{\vec{r}}$, which, in turn, implies that the vector $\vec{A}(t)$ should evolve in a fixed plane, orthogonal to $\vec{L}$.

On the other hand, in the case of rapid evolution we can also write

$$
\frac{\dot{A}_{i} \dot{A}_{j}}{a^{2}} \delta^{i j} \approx(\dot{\vec{r}})^{2}=\dot{r}^{2}+\frac{L^{2}}{r^{2}} .
$$

Thus, the total energy density can be written as

$$
\rho=\frac{1}{2} \frac{\dot{A}_{i} \dot{A}_{j}}{a^{2}} \delta^{i j}+V\left(A^{2}\right)=\frac{1}{2} \dot{r}^{2}+\frac{L^{2}}{2 r^{2}}+V\left(-r^{2}\right),
$$

which can be considered as constant within the short time scale of variation of the vector field.

Thus, we are left with the reduced radial problem, i.e., the motion of a particle in the radial dimension in the presence of the effective potential

$$
V_{\mathrm{eff}}(r)=\frac{L^{2}}{2 r^{2}}+V\left(-r^{2}\right)
$$

where the value of the constant $L$ is set by the initial conditions. The other constant of motion associated with this problem is the energy density $\rho_{0}=V_{m}$.

\footnotetext{
${ }^{1}$ There is another possible solution: $V^{\prime}\left(A^{2}\right)=0$. In this case, the isotropy theorem cannot be applied because the evolution of $A_{i}$ is not rapid. It can be showed that $\dot{A}_{i}=C_{i} / a$, and the anisotropic components of the EMT decay as $a^{-4}$ [15].
} 
As commented before, if the temporal evolution of $A_{i}$ is sufficiently rapid and we concentrate only in a time interval of order $\omega_{i}^{-1}$, we can ignore the effect of the universe expansion, i.e., we can consider $a$ as constant. In this case, we can write the equation of motion for the vector field as $\ddot{A}_{i}=2 V^{\prime}\left(A^{2}\right) A_{i}$. Let us define

$$
G_{i j}=\frac{\dot{A}_{i} A_{j}}{a^{2}}, \quad i, j=1,2,3,
$$

and take its time derivative:

$$
\dot{G}_{i j}=\frac{\ddot{A}_{i} A_{j}}{a^{2}}+\frac{\dot{A}_{i} \dot{A}_{j}}{a^{2}} .
$$

Using the equations of motion, we get

$$
\dot{G}_{i j}=2 V^{\prime}\left(A^{2}\right) \frac{A_{i} A_{j}}{a^{2}}+\frac{\dot{A}_{i} \dot{A}_{j}}{a^{2}} .
$$

Integrating this expression in a given time interval $[0, T]$,

$$
\frac{G_{i j}(T)-G_{i j}(0)}{T}=\left\langle 2 V^{\prime}\left(A^{2}\right) \frac{A_{i} A_{j}}{a^{2}}\right\rangle+\left\langle\frac{\dot{A}_{i} \dot{A}_{j}}{a^{2}}\right\rangle,
$$

with $i=1,2,3$. If the motion is periodic and $T$ corresponds to the oscillation period, the left-hand side (1.h.s.) vanishes. If the motion is not periodic, but $A_{i}$ and $\dot{A}_{i}$ are bounded by taking $T$ sufficiently large, but satisfying $H^{-1} \gg T \gg \omega_{i}^{-1}$ for any $i$, we can also neglect the 1.h.s. of the equation. In those cases, we have

$$
\left\langle\frac{\dot{A}_{i} \dot{A}_{j}}{a^{2}}\right\rangle=-\left\langle 2 V^{\prime}\left(A^{2}\right) \frac{A_{i} A_{j}}{a^{2}}\right\rangle, \quad i, j=1,2,3 .
$$

By using this result in Eq. (11), we straightforwardly get

$$
\left\langle T^{i}{ }_{j}\right\rangle=0, \quad i \neq j,
$$

i.e., the average EMT is diagonal. Also, using Eq. (22) in Eq. (9), we obtain

$$
\left\langle p_{k}\right\rangle \equiv-\left\langle T^{k}{ }_{k}\right\rangle=\left\langle\frac{1}{2} \frac{\dot{A}_{i} \dot{A}_{j}}{a^{2}} \delta^{i j}\right\rangle-\left\langle V\left(A^{2}\right)\right\rangle, \quad k=1,2,3,
$$

i.e., all the average pressures are the same, so that the isotropy of the mean value of the EMT is proved:

$$
\left\langle T^{i}{ }_{j}\right\rangle=-\langle p\rangle \delta^{i}{ }_{j} .
$$

It is interesting to note that the virial relations Eq. (22) also allow us to get the average equation of state for a power-law potential: $V=\lambda\left(A_{\mu} A^{\mu}\right)^{n}$. In this case, we have

$$
\left\langle\frac{1}{2} \frac{\dot{A}_{i} \dot{A}_{j}}{a^{2}} \delta^{i j}\right\rangle=n\left\langle V\left(A^{2}\right)\right\rangle
$$

which implies that

$$
\left\langle p_{k}\right\rangle=\langle p\rangle=(n-1)\left\langle V\left(A^{2}\right)\right\rangle, \quad k=1,2,3 .
$$

On the other hand, for the average energy density, we get from Eq. (8)

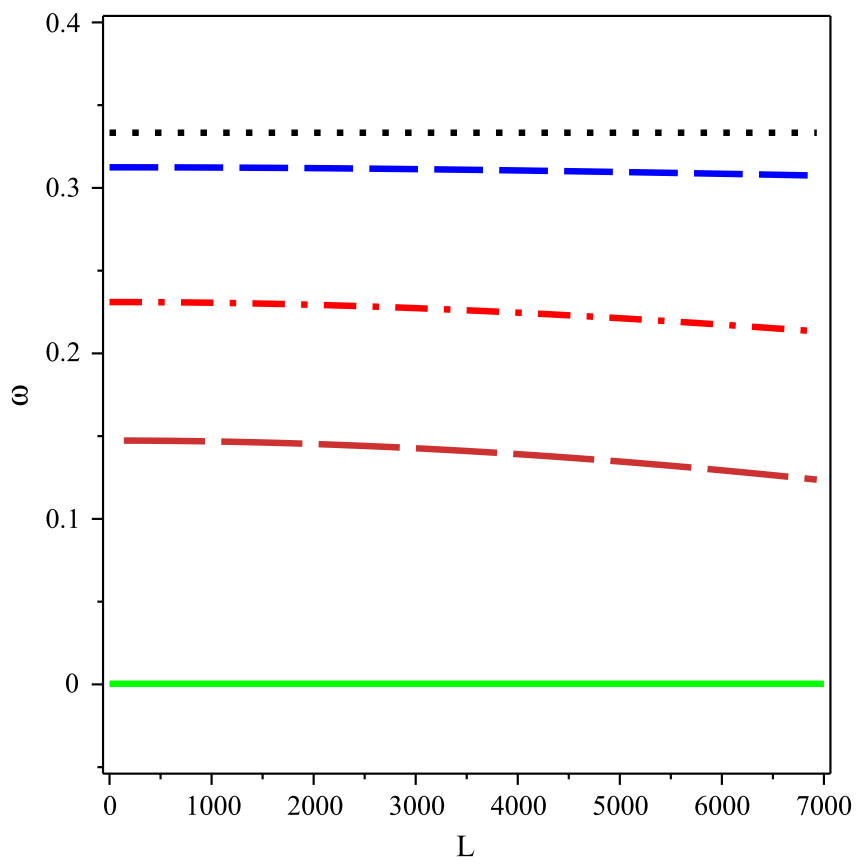

FIG. 1 (color online). The effective equation of state $\omega \equiv$ $\langle p\rangle /\langle\rho\rangle$ can be computed numerically by integrating Eqs. (8) and (9) with respect to the $r$ variable with the use of Eqs. (15) and (16). Thus, in the case $V=-a A^{2}, \omega=0$ and the coherent vector oscillations behave as cold dark matter (CDM); or, for $V=b A^{4}, \omega=1 / 3$ and they behave as radiation. These results agree with Eq. (29). For a general potential, the effective equation of state depends on the initial conditions: $V_{m}$ and $L$. In this figure, one can see the $L$ dependence of $\omega$ for $V=-a A^{2}+b A^{4}$ and $V_{m}=10^{4}$. Depending on the particular values of $a$ and $b$, the equation of state interpolates between the radiation and the CDM behavior: from top to bottom respectively, the dotted (black) line corresponds to $(a, b)=\left(10^{-7}, 2.4\right.$. $\left.10^{-7}\right)$, blue (dashed) line corresponds to $(a, b)=\left(0.1,2 \cdot 10^{-4}\right)$, dashed-dotted (red) for $(a, b)=\left(0.4,10^{-4}\right)$, long dashed (orange) for $(a, b)=\left(0.7,6 \cdot 10^{-5}\right)$ and continuous (green) for $(a, b)=\left(10^{-2}, 10^{-11}\right)$.

$$
\langle\rho\rangle=(n+1)\left\langle V\left(A^{2}\right)\right\rangle,
$$

and finally,

$$
\omega=\frac{\langle p\rangle}{\langle\rho\rangle}=\frac{n-1}{n+1},
$$

which agrees with the scalar case [5] and shows that the average equation of state does not depend on the polarization of the vector oscillations. (Notice that the scalar case corresponds to $L=0$, i.e., the evolution of a particle in one spatial dimension.) In the case of a general potential not necessarily of the power-law form, the equation of state cannot be obtained analytically and, in general, it will depend on the initial conditions for the vector oscillations, as Fig. 1 shows. Notice that the average energy density and pressure satisfy the conservation Eq. (12) up to corrections of order $\mathcal{O}(H T)$. 
We would think that the isotropy of the average EMT could have been inherited from the isotropy of the FLRW metric. But repeating the process for a Bianchi I metric

$$
d s^{2}=d t^{2}-a_{1}^{2}(t) d x^{2}-a_{2}^{2}(t) d y^{2}-a_{3}^{2}(t) d z^{2},
$$

if we replace

$$
\frac{A_{i}}{a} \rightarrow \frac{A_{i}}{a_{i}}, \quad i=1,2,3,
$$

and assume $\omega_{i}$ much greater than $H_{i}=\dot{a}_{i} / a_{i}$, it is straightforward to obtain the same results for $\left\langle T^{\mu}{ }_{\nu}\right\rangle$.

The previous results can be directly extended to general spacetime geometries (not necessarily homogeneous). With that purpose, let us consider a locally inertial observer at $x_{0}^{\mu}=0$ and write the metric tensor in Riemann normal coordinates around $x_{0}^{\mu}[16]$ :

$$
g_{\mu \nu}(x)=\eta_{\mu \nu}+\frac{1}{3} R_{\mu \alpha \nu \beta} x^{\alpha} x^{\beta}+\ldots
$$

Let us assume that the following conditions hold:

(1) The Lagrangian of the vector field is restricted to be given by the form of Eq. (2).

(2) The vector field evolves rapidly:

$$
\begin{gathered}
\left|R_{\lambda \mu \nu}^{\gamma}\right| \ll \omega_{i}^{2}, \quad \text { and }\left|\partial_{j} A_{i}\right| \ll\left|\dot{A}_{i}\right|, \\
i, j=1,2,3,
\end{gathered}
$$

for any component of the Riemann tensor.

(3) $A_{i}$ and $\dot{A}_{i}$ remain bounded in the evolution.

The second condition implies that if we are only interested in time scales of order $\omega_{i}^{-1}$, then we are in a normal neighborhood and we can neglect the second term in Eq. (32) and can also work with a homogeneous vector field. In such a case, it is possible to rewrite all the above equations with $a=1$. Thus, by using an interval $[0, T]$ that verifies the condition

$$
\left|R_{\lambda \mu \nu}^{\gamma}\right| \ll T^{-2} \ll \omega_{i}^{2}
$$

for any of the components of the Riemann tensor and any of the spatial components of the vector $(i=1,2$ and 3$)$, it is possible to obtain Eq. (21) and prove that the mean value of the EMT is isotropic. It is interesting to note that if the metric is nonhomogeneous, the EMT can be nonhomogeneous, but its average value will be isotropic as seen from a locally inertial frame.

In this work, we have considered the evolution of general Abelian vector fields in an expanding universe. We have shown by means of a mechanical analogy with a particle moving in a central potential that a generalized version of the virial theorem ensures that, for rapid dynamics, the average EMT is always diagonal and isotropic, despite the fact that the evolution always takes place in a fixed plane. The result can be extended to arbitrary geometries within a normal neighborhood of any locally inertial observer.

This work has been supported by MICINN (Spain) under Project Nos. FIS 2008-01323, FIS2011-23000, FPA201127853-01 and Consolider-Ingenio MULTIDARK under Contract No. CSD2009-00064.
[1] T. Damour and V. F. Mukhanov, Phys. Rev. Lett. 80, 3440 (1998); A. R. Liddle and A. Mazumdar, Phys. Rev. D 58, 083508 (1998).

[2] R. D. Peccei and H. R. Quinn, Phys. Rev. Lett. 38, 1440 (1977); Phys. Rev. D 16, 1791 (1977); L. F. Abbott and P. Sikivie, Phys. Lett. 120B, 133 (1983).

[3] B. de Carlos, J. A. Casas, F. Quevedo, and E. Roulet, Phys. Lett. B 318, 447 (1993); M. Gasperini and G. Veneziano, Phys. Rev. D 50, 2519 (1994); J. A. R. Cembranos, Phys. Rev. Lett. 102, 141301 (2009); J. Phys. Conf. Ser. 315, 012004 (2011).

[4] J. A. R. Cembranos, A. Dobado, and A. L. Maroto, Phys. Rev. D 65, 026005 (2001); Phys. Rev. Lett. 90, 241301 (2003); Phys. Rev. D 68, 103505 (2003); A. L. Maroto, Phys. Rev. D 69, 043509 (2004); Phys. Rev. D 69, 101304 (2004).

[5] M. S. Turner, Phys. Rev. D 28, 1243 (1983).

[6] A. R. Liddle and R. J. Scherrer, Phys. Rev. D 59, 023509 (1998); S. Dutta and R. J. Scherrer, Phys. Rev. D 78, 083512 (2008).

[7] A. Golovnev, V. Mukhanov and V. Vanchurin, J. Cosmol. Astropart. Phys. 06 (2008) 009; T. Koivisto and D. F.
Mota, J. Cosmol. Astropart. Phys. 08 (2008) 021; K. Bamba, S.'i. Nojiri, and S.D. Odintsov, Phys. Rev. D 77, 123532 (2008); B. Himmetoglu, C. R. Contaldi, and M. Peloso, Phys. Rev. Lett. 102, 111301 (2009); A.E. Gumrukcuoglu, B. Himmetoglu, and M. Peloso, Phys. Rev. D 81, 063528 (2010).

[8] L. H. Ford, Phys. Rev. D 40, 967 (1989).

[9] C. Armendariz-Picon, J. Cosmol. Astropart. Phys. 07 (2004) 007; C. G. Boehmer and T. Harko, Eur. Phys. J. C 50, 423 (2007).

[10] J. Beltran Jimenez and A.L. Maroto, Phys. Rev. D 78, 063005 (2008); J. Beltran Jimenez and A.L. Maroto, J. Cosmol. Astropart. Phys. 03 (2009) 016; J. Beltran Jimenez and A.L. Maroto, Phys. Lett. B 686, 175 (2010); E. Carlesi, A. Knebe, G. Yepes, S. Gottloeber, J. Beltran Jimenez, and A. L. Maroto, Mon. Not. R. Astron. Soc. 418, 2715 (2011).

[11] J. Redondo and M. Postma, J. Cosmol. Astropart. Phys. 02 (2009) 005.

[12] K. Dimopoulos, Phys. Rev. D 74, 083502 (2006).

[13] A. E. Nelson and J. Scholtz, Phys. Rev. D 84, 103501 (2011). 
[14] H. K. EriksenF. K. Hansen, A. J. Banday, K. M. Górski, and P. B. Lilje, Astrophys. J. 605, 14 (2004); , , 609, 1198 (E) (2004); G. Hinshaw et al. (WMAP Collaboration), Astrophys. J. Suppl. Ser. 170, 288 (2007); K. Land and J. Magueijo, Phys. Rev. Lett. 95, 071301 (2005); A. Kashlinsky F. Atrio-Barandela, D. Kocevski, and H.
Ebeling, Astrophys. J. Lett. 686, L49 (2008); R. Watkins, H. A. Feldman, and M. J. Hudson, Mon. Not. R. Astron. Soc. 392, 743 (2009).

[15] J. A. R. Cembranos et al. (work in progress).

[16] A.Z. Petrov, Einstein Spaces (Pergamon, Oxford, 1969). 